\title{
Statistical Characterization of High Angle Graphene Grain Boundaries at Atomic Resolution
}

\author{
Colin Ophus $^{1}$, Haider Rasool ${ }^{2,3}$, Alex Zettl ${ }^{2,3}$, Michael F Crommie ${ }^{2,3}$ and Ulrich Dahmen ${ }^{1}$ \\ 1. National Center for Electron Microscopy, Lawrence Berkeley National Laboratory, Berkeley, USA \\ 2. Department of Physics, University of California Berkeley, Berkeley, USA \\ 3. Materials Science Department, Lawrence Berkeley National Laboratory, Berkeley, USA
}

The mechanical strength [1] and electronic properties [2] of crystalline materials often depends quite strongly on the location and structure of defects in the crystalline matrix. Graphene is no different, despite having a two-dimensional crystal structure; many deposition methods produce polycrystalline films containing a high density of grain boundaries (GBs) [3]. In this work, we measure the structure of high-angle grain boundaries in graphene grown on copper substrates, with many different disorientations angles up to and including $30^{\circ}$.

We have collected a large number of HRTEM micrographs to provide good statistics for the local configuration at the grain boundaries. Strain measurements on representative boundaries were performed on exit wave reconstructions from focal series, because of the greatly improved signal-tonoise and measurement precision. Exit wave reconstructions were performed using the algorithms described in [4], along with a new method for numerical aberration correction in crystal lattices. These new high-precision strain measurements are compared to our previous work on similar boundaries [5].

A semi-automated analysis routine was developed to map out the structure of the graphene GBs from HRTEM micrographs. Figure 1 shows a few of the steps including (from left to right) the raw micrograph, correction of the monochromated illumination intensity falloff, filter detection of the dual lattice and automated lattice detection and fitting. By using automation we are able to greatly increase the number of micrographs analyzed. We have chosen the dual lattice to graphene because of the simpler crystallographic basis (one site instead of two). Figure 2 shows some examples of GB configuration that have been experimentally measured. Red cells mark rings containing 5 carbon atoms while blue cells show 7-member carbon rings.

From this data we can understand the local structure of high angle graphene GBs and evaluate the accuracy of various theoretically predicted configurations in the literature. Most of the observed boundaries span the range of disorientations from 21 to $30^{\circ}$ around special coincidence site lattice positions such as Sigma 7 and Sigma 13. Our experiments allow us to correlate the observed local defect configurations and boundary roughness with the deviation from the exact disorientations predicted for the coincidence site lattices [6,7].

\section{References}

[1] Y Wei, J Wu, H Yin, X Shi, R Yang and M Dresselhaus, Nature Materials 11 (2012) p. 759

[2] OV Yazyev and SG Louie, Nature Materials 9 (2010) p. 806

[3] E Cockayne, Physical Review B 85 (2012) 125409

[4] C Ophus and T Wealds, Ultramicroscopy 113 (2012) p. 88

[5] H Rasool, C Ophus, WS Klug, A Zettl and JK Gimzewski, Nature Comm. 4 (2013) p. 2811

[6] KC Lee and U Erb, Beilstein Journal of Nanotechnology 4 (2013) p. 292 
[7] The authors acknowledge the financial support of the Office of Science, Office of Basic Energy Sciences of the US Department of Energy under contract number De-AC02-05CH11231.

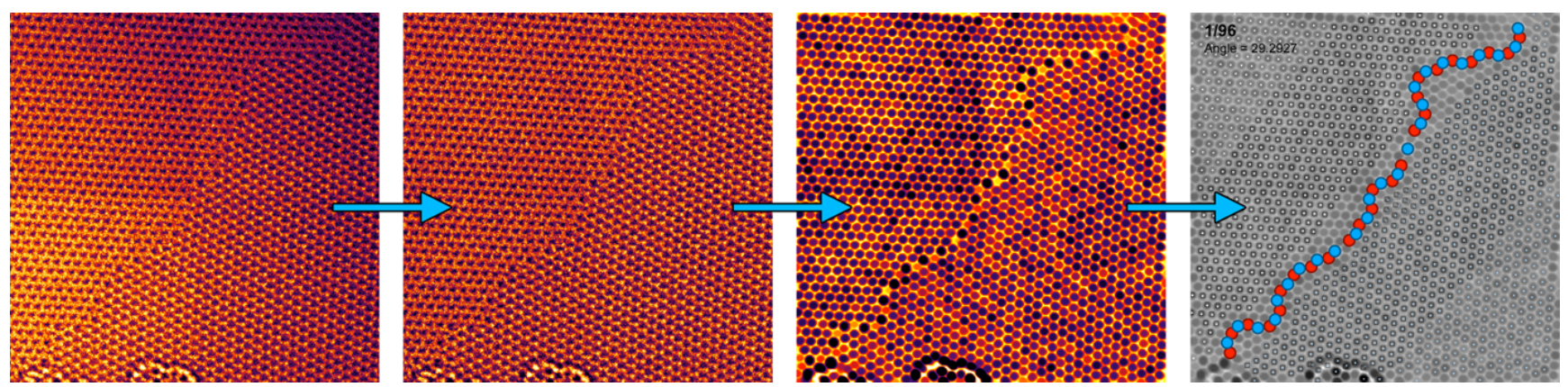

Figure 1. Some steps of the semi-automated analysis routine for mapping grain boundary structures.
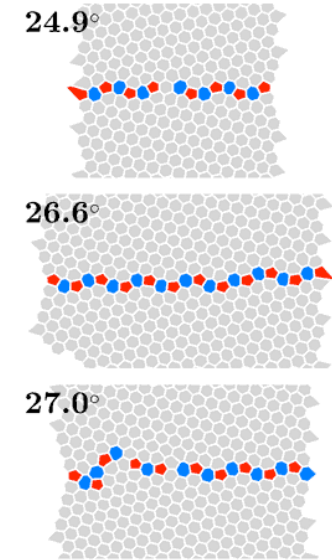

$28.0^{\circ}$

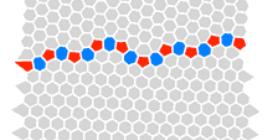

$28.5^{\circ}$
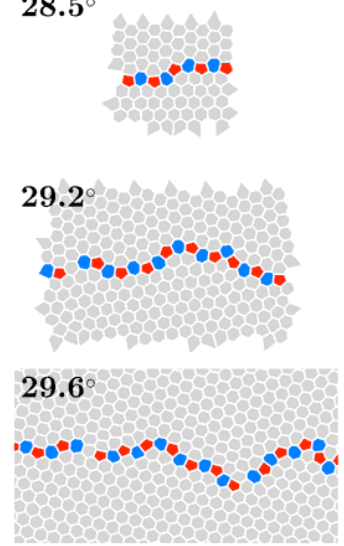
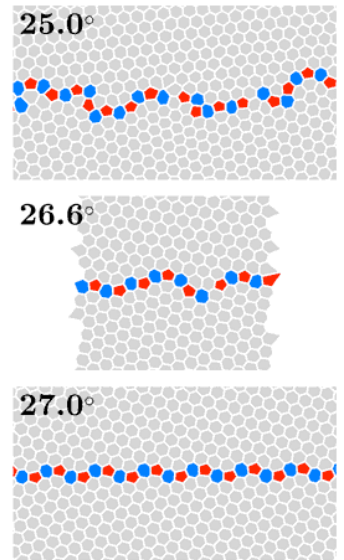

28.3

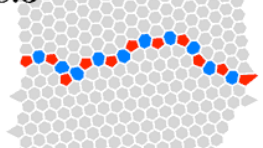

$28.8^{\circ}$

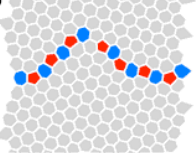

20.2
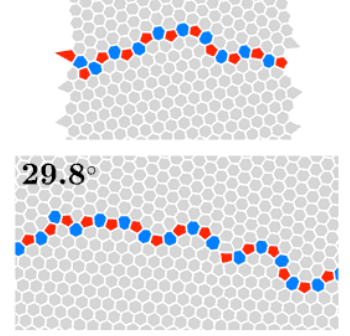
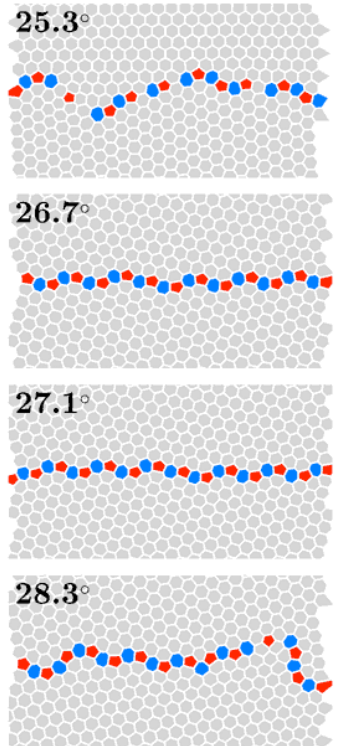

$28.9^{\circ}$

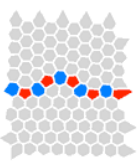

$29.3^{\circ}$
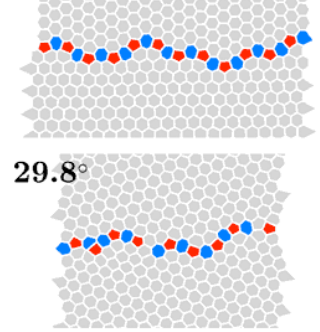
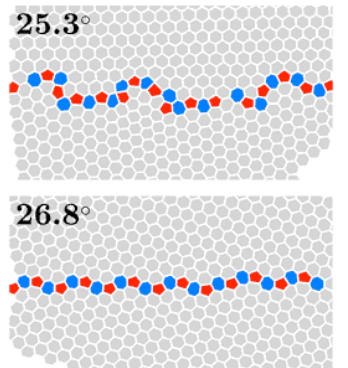

$27.1^{\circ}$

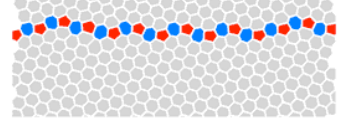

$28.5^{\circ}$

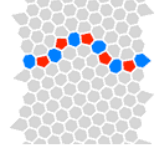

$29.1^{\circ}$

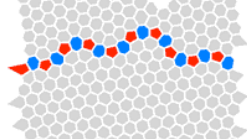

$29.3^{\circ}$

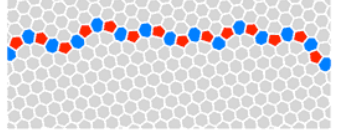

$29.8^{\circ}$

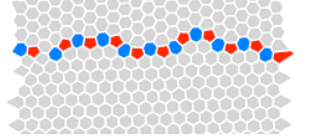

$25.5^{\circ}$
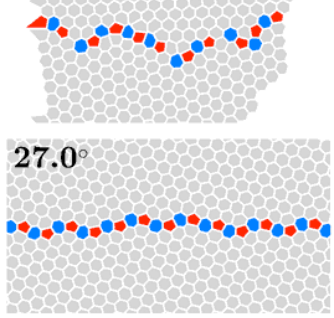

$27.5^{\circ}$

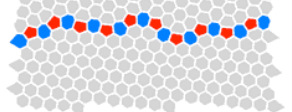

$28.5^{\circ}$

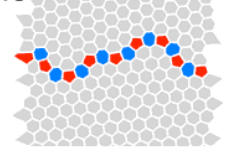

29.1

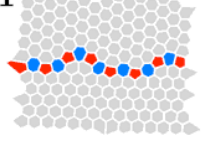

$29.4^{\circ}$

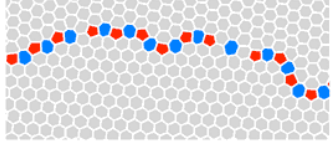

$29.9^{\circ}$

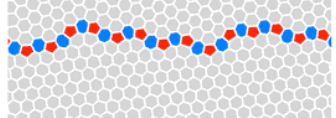

Figure 2. Some examples of high-angle graphene grain boundary configurations, with lowest disorientation angle given for each boundary. Note that the disorientation angles might be slightly incorrect due to tilt of the graphene foil with respect to the electron beam axis. 